\title{
PILAR: Sharing VISIR Remote Labs Through a Federation
}

\author{
Felix Garcia-Loro, Elio San Cristobal; \\ Gabriel Diaz; Manuel Castro \\ UNED \\ Madrid, Spain \\ \{fgarcialoro; elio; gdiaz; \\ mcastro\}@ieec.uned.es \\ Andre Fidalgo; Gustavo Alves \\ IPP \\ Oporto, Portugal \\ \{anf; gca\}@isep.ipp.pt \\ Michael Auer
IAOE
Wien, Austria
auer@cti.ac.at
}

\author{
Pablo Orduña \\ DeustoTech-LabsLand \\ Bilbao, Spain \\ pablo@labsland.com
}

Unai Hernandez-Jayo; Javier Garcia-

Zubia

Deusto

Bilbao, Spain

\{unai.hernandez; zubia\}@deusto.es

Carla Garcia; Ricardo Tavio

$$
\text { EVM }
$$

Grand Canary, Spain

\{carla.garcia; ricardo.tavio\}@evm.net

\author{
Wlodek Kulesza; Kristian Nilsson \\ BTH \\ Karlskrona, Sweden \\ \{wlodek.kulesza; \\ kristian.nilsson\}@bth.se \\ Christian Kreiter; Andreas Pester \\ CUAS \\ Villach, Austria \\ \{c.kreiter; pester\}@fh-kaernten.at \\ Kati Valtonen; Elina Lehtikangas \\ OMNIA \\ Espoo, Finland \\ $\{$ kati.valtonen \\ elina.lehtikangas\}@omnia.fi
}

\begin{abstract}
Social demands have promoted an educational approach based on an "anywhere and anytime" premise. Remote laboratories have emerged as the answer to the demands of technical educational areas for adapting themselves to this scenario. The result has not only benefit distance learning students but has provided new learning scenarios both for teachers and students as well as allowing a flexible approach to experimental topics. However, as any other solution for providing practical scenarios (hands-on labs, virtual labs or simulators), remote labs face several constraints inherited from the subsystems of its deployment - hardware (real instruments, equipment and scenario) and software (analog/digital conversions, communications, workbenches, etc.)- This paper describes the Erasmus+ project Platform Integration of Laboratories based on the Architecture of visiR (PILAR) which deals with several units of the federation installed in different educational institutions and devoted to analog electronics and electrical circuits. Based on the limitations of remote labs, the need for the federation will be justified and its benefits will be described.
\end{abstract}

Keywords-VISIR, federation; remote lab, electronics remote lab.

\section{INTRODUCTION}

A VISIR system replicates a hands-on analog electronics lab on a virtual workbench and it is based on a switching matrix relay for providing users with a flexible experimental environment. It allows students to monitor signals, acquire measures, design electronics circuits and, essentially, control remotely an electronics lab. The adaptability and reliability of VISIR system have been described before: it has been integrated into different learning scenarios and at different educational levels [5], providing in all of them satisfactory results.

PILAR project [1] $<<$ Erasmus + Strategic Partnership $n^{\circ}$ 2016-1-ES01-KA203-025327: Platform Integration of Laboratories based on the Architecture of visiR $>>$ started up in 2016 with the aims of enhancing practical instructive scenarios- in particular electronics practical experimentation- at different educational levels-- from lower secondary students to PhD master students-- through remote labs -specifically through VISIR remote laboratory - and improving the repository, efficiency and accessibility of these educational resources. PILAR project also holds a connection with the 2013 "Communication on Opening Up Education" [1], according to some of its main objectives:

- The creation of opportunities for innovative approaches: PILAR consortium makes possible new learning/teaching scenarios providing flexible experiments in a manner that a single VISIR system, working in island mode, cannot reach.

- The increase in the use of Open Educational Resources (OER): PILAR consortium enhances the reusability and exchange of the educational resources based on the VISIR system among the VISIRpartners (partners with a VISIR system). Furthermore, through the designed framework for the consortium -and once established the policies to become a consortium member and the service level agreement (SLA) type for each of the remote lab services offered-, the federation will be completely opened to other partners in Europe, through easy gateways to the federation, allowing to extend the capabilities of PILAR to interested educational institutions.

The federation involves 5 higher education institutions from Spain, Sweden, Portugal and Austria $<<$ Universidad Nacional de Educación a Distancia, (UNED), Universidad de Deusto (UDEUSTO), Blekinge Tekniska Hogskola (BTH), Instituto Politecnico do Porto (IPP) and Fachhochschule Kärnten (CUAS) $>>$ each of them providing a VISIR system, educational resources and a broad experience in using VISIR in different learning environments. Together with the aforementioned higher educational institutions, PILAR consortium is composed of the Internationale Gesellschaft fur Online Engineering Verein (IAOE); a non-profit organization from Austria focused on the application, spreading and development of online engineering; Ecosistemas Virtuales y Modulares SL (EVM) a small/medium sized enterprise from Spain with wide experience in using online training as a tool for improving 
the professional skills; and the Espoon Seudun Koulutuskuntayhtyma Omnia (OMNIA), a multi-sector education provider from Finland centered on upper secondary vocational education and training, as well as apprenticeship training for young people and adults.

This paper is structured as follows: Section II presents an overview of the VISIR system; Section III provides the challenges faced so far and the technical solutions implemented. Section IV presents the next steps and conclusions.

\section{VISIR REMOTE LAB}

The VISIR remote lab was developed at BTH (by the Department of Signal Processing) under a project started in 2006 and also named VISIR [3]. A VISIR remote lab consists of a hardware system at the side of the providing institution and a software coating at both sides $<<$ student and institution $>>$. Additionally, it requires real components, their description files and the physical connections to be allowed among them.

The hardware and software in VISIR has been widely described [3][4],

\section{A. Hardware}

As we underlined before, the hardware in a VISIR system can be classified as:

- Instrumentation hardware: The hardware required for the remote lab to be operational independent of the circuits/components to experiment with. Instrumentation hardware is arguably the instruments and equipment in a traditional electronics laboratory in - digital multimeter, oscilloscope, power supply, function generator and breadboard.

- Experimentation hardware: The specific hardware required for the construction and designing of the circuits, which consists of a set of components depending on the desired experiment.

\section{1) Instrumentation Hardware}

A VISIR system requires, at least, the following elements in order to be a functional electronics laboratory:

a) Instrumentation platform: The instrumentation platform consists of a chassis which houses the Peripheral Component Interconnect (PCI) eXtensions for Instrumentation (PXI) boards from National Instruments, NI, [4]. Each board is a lab instrument. It also is in charge of the communications, power, cooling. In the case of the

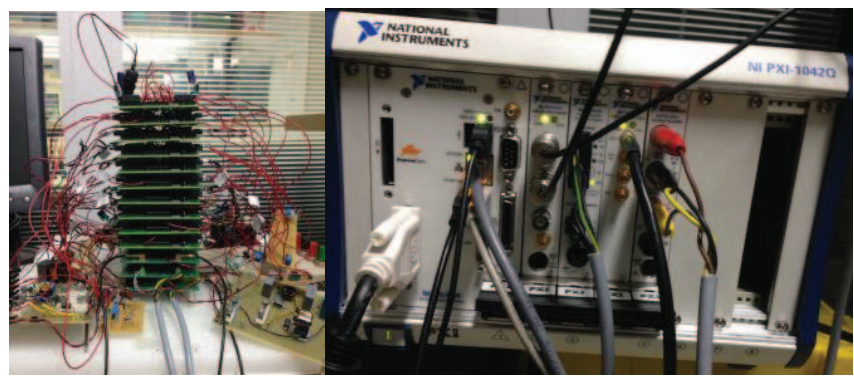

Fig. 1. VISIR system at UNED: PXI chasis containing the instruments and equipment cards from NI (right); relay switching matrix installed at UNED, consisting on 3 instrument/equipment boards and 10 component boards.
VISIR system shown in figure 1, the chassis also houses the controller $-\mathrm{a}$ PC - . However, this can be an external unit using the appropriate communications interface. As in a typical electronics hands-on lab, VISIR must count at least with a function generator, power supply, multimeter and oscilloscope. Replicated instruments is an option -i.e. a VISIR system may handle 2 multimeters-

b) Relay switching matrix: The relay switching matrix acts as the breadboard of the traditional hands-on lab. It is a stack of 4 types of boards -DMM board, oscilloscope board, source board and components board- Each board has a controller and the source board houses the controller of the matrix. The communications between the relay switching matrix and the instrumentation controller are carried out by means of a USB cable and a controller housed in the source board. A typical VISIR system has an oscilloscope board, a source board, 1 or 2 DMM boards and from 4 to 10 components boards. The VISIR system shown in figure 1 counts with 10 components boards.

\section{2) Experimentation Hardware}

The experimentation hardware are the components and their connections in the matrix of nodes in the relay switching matrix. In figure 1some external circuits surrounding the relay switching matrix are clearly perceptible. Figure 2 shown a component board with an external circuit., this figure also shows the matrix of nodes $<<$ A to I and X1 to X6 $>>$ with the nodes propagation strip in the middle, the component and relays placement at the board.

\section{B. Software}

The software required in a VISIR node can be divided into the required software to provide an operational electronics remote lab to control it and experiment with it, and the required configuration and description files required for the experiments and installed components. Meanwhile this required software is common to all the VISIR systems and the different versions of the measurement server or client are valid for all the VISIR nodes, as in the case of the hardware the particularity of the experiments designed at each VISIR node turns each system different from one to each other: as if they were different labs focused on different experiments.

- Experiment client: The experiment client consists of a simulated workbench where the user interacts with

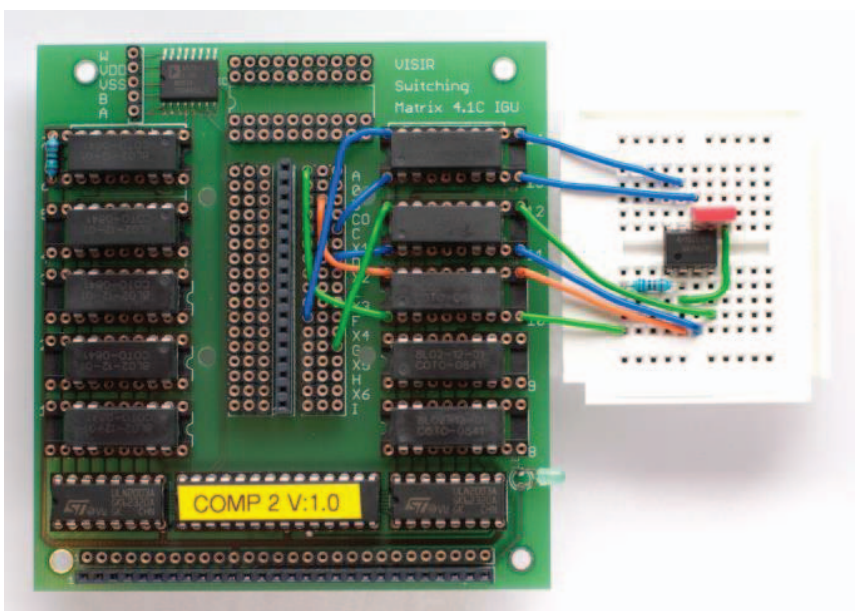

Fig. 2. Component board with an external circuit. 
the different instruments, configure them, and from where the student can design the experiments and send them to measurement server to be collated before being physically constructed. In short, is where the student designs the experiment.

- Measurement server. The measurement server receives the user requests to control the lab. It checks the user's authentication, manages the queue of users and collates the designed circuit by the user with the allowable circuits included in the max files previously designed by the teacher.

- Equipment server: The equipment server is responsible for configuring the instruments and constructing physically the circuits according to the validated request provided by the measurement server.

\section{A JOURNEY FROM AN ISLAND TO A COMMUNITY}

The need for a federation of VISIR systems has been dealt with previously by the consortium [10]-[13]. The main reasons are based on scalability, availability and economical optimization:

- Constructive limitations about the number of component boards allowable in a relay switching matrix.

- Constructive limitations in the system about the number of allowable components.

- Constructive and designing limitations about the allowable connections.

- Service availability.

- Economic factors regarding qualified personnel, physical installations, etc.

According to the project requirements, first PILAR lesson repository must:

- R1: Enable the access of teachers and students to all the PILAR VISIR systems.

- R2: The solution must be federated, meaning that teachers and students will not need to be registered in each of the VISIR systems of the PILAR consortium.

- R3: Students and teachers do not need to be aware of which VISIR system they are using. They should be able to move from one system to other without knowing what VISIR system supports what.

- R4: Teachers must be able to try the lessons without having their own infrastructure.

- R5: Teachers must be able to use the contents without having their own infrastructure.

\section{A. Island}

Although the VISIR systems provided by the partners (UNED, BTH, Deusto, IPP, CUAS) are based on the same architecture and the installed hardware (instrumentation hardware) is compatible among the consortium VISIR systems, the software layer in which each partner relies on differs one from each other. Each of them uses different settings for management and access, which is relevant to the PILAR lesson repository:

- UNED: the VISIR system is managed through a software called WebLab-Deusto. However, on top of that, UNED uses a proprietary reservations component that guarantees that in some cases, only one person at a time can access the VISIR system (and the rest of the time, up to 30 concurrent students access the system). This reservations component supports the IMS LTI protocol.

- ISEP IPP uses WebLab-Deusto for managing users.

- UDEUSTO: the VISIR system uses WebLab-Deusto for managing the access to the VISIR laboratory.

- BTH: OpenLabs is the software in charge of managing their VISIR system.

- CUAS: Their VISIR system relies on a custom software called e-dispatcher.

\section{B. Community}

Moodle is an Open Source Learning Management System that supports many of the features required for the first version of the PILAR lesson repository: it supports IMS LTI, which is a protocol that both gateway4labs and the reservation mechanism of UNED support.

\section{Pilot phase}

The design of the technical solution for the PILAR lesson repository is shown in figure 3 . On it, lessons can be placed in a Moodle system. Each lesson can use one VISIR system, but the technical solution to access it is always the same (IMS LTI) and students can use any VISIR of the consortium in a transparent way (requirement R1). Students do not need to register in each platform (requirement R2). PILAR places lessons in Moodle already linked to a particular VISIR, but students do not need to be aware of which VISIR they need to use (requirement R3). Teachers can register in Moodle using Moodle tools for registration without having their own infrastructure (requirement R4). And teachers and students can register so students can be sent to different lessons to use the equipment without teachers owning a different infrastructure (requirement R5).

\section{PILAR web-repository}

The PILAR web-repository is being developed. The structure will include the information shown in figure 4. Depending on the user profile (admin, consortium partner, VISIR partner, developer partner or external teacher) permissions will differ (i.e. external partner will be allowed to download all the documents related to the experiment but nothing about the configuration in VISIR). from it, the consortium will have the required tools to articulate the different actors around the community.

This web must be deployed for the second phase of the project. From it, any external teacher will be available to pick up the desired experiment and include them in his own/institutional LMS/CMS. To open a course in PILARMoodle for those external teachers without an LMS/CMS compatible with LTI protocol is available too. 


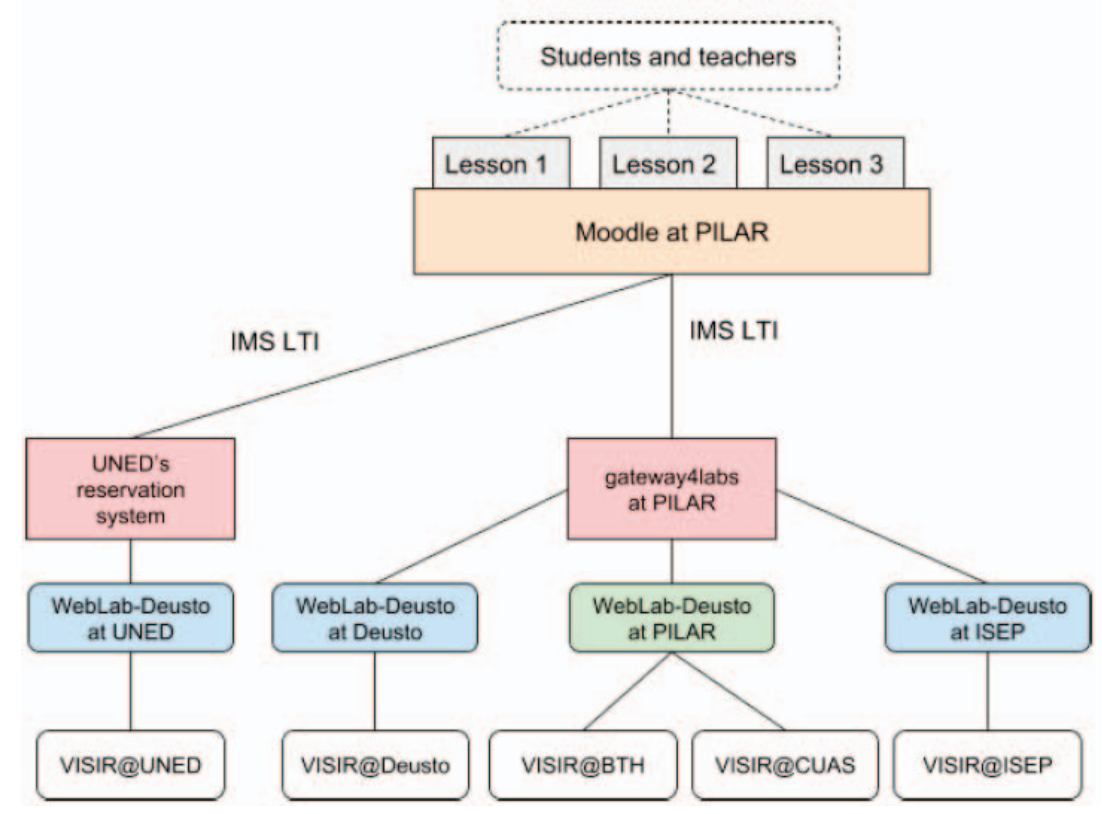

Fig. 3. Example of max file at VISIR system 1 and VISIR system2.

\section{CONCLUSIONS AND FUTURE WORK}

The pilot course counts with 15 different experiments. At least 1 experiment has been provided by each VISIR provider from the consortium. So far, 206 users are enrolled in the platform. 112 of them are enrolled in PILAR pilot course, whereas the other 94 students are from an electronics subject at UNED, who have used a practical course based on VISIR remote lab, in PILAR platform as a mandatory task.

Apparently, several circuits are common for 2 or more VISIR nodes from the consortium — voltage divider using 1 $\mathrm{k} \Omega$ and $10 \mathrm{k} \Omega$ resistors or a half-way rectifier using a $1 \mathrm{~N} 4007$ rectifier diode and a $10 \mathrm{k} \Omega$ resistor-, However, some minor differences in the matrix (experimentation hardware) or in the files (max files) turns these experiments into different scenarios. These different configurations for a VISIR system can be equivalent in some subset of experiments, but not for the whole spectrum of them. i.e. the $\max$ files from figure 5 are equivalent for some measurements of a Ucc - $10 \mathrm{k} \Omega-1 \mathrm{k} \Omega-$ GND voltage divider: voltage drop at any resistor, Amperemeter between Ucc and $10 \mathrm{k} \Omega$ and between $1 \mathrm{k} \Omega$ and GND, both configurations can use the function generator, etc.; However, max file from VISIR system 1 allows Ucc $-1 \mathrm{k} \Omega-10 \mathrm{k} \Omega$ - GND whereas VISIR system2 doesn't, and VISIR system 2 allows to connect the amperemeter between both

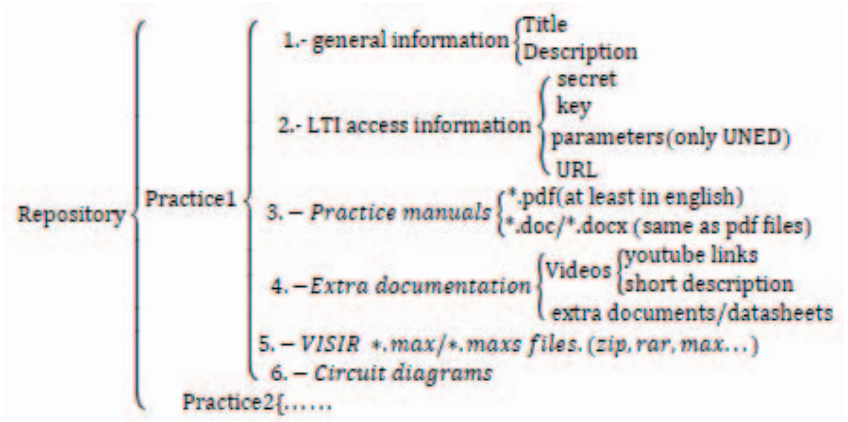

Fig. 4. OER, practices repository in PILAR resistors while in VISIR system1 it is physically unviable (both resistors are rigidly connected at node D).

Obviously, the resulting arrangement of the components in the relay switching matrix, the files describing the components installed and the allowable connections can be replicated. However, each VISIR-provider in the consortium has designed the matrix according to his particular educational needs.

In the next phase, the federation must probe the flexibility of the system for a balanced experimentation of the users among the PILAR consortium. The aforementioned equivalent circuits must be strategically defined.

The following features must be covered in the future version of the PILAR lesson repository:

- FR1: Support of distributed load balancing: if a circuit is available in more than one VISIR system, students should be dynamically redirected to one or

\begin{tabular}{|llll|}
\hline & file.max @VISIR1 & \\
VFGENA_FGENA1 & A 0 & $\max : 5$ & \\
VDC+25V_4 & B & max:15 & imax:0.5 \\
VDCCOM_24_2 & & 0 & \\
& & & \\
SHORTCUT_1_5 & A B & \\
SHORTCUT_1_5 & B E & \\
SHORTCUT_1_5 & B C & 10K \\
R_3_6 & C D & 1K \\
R552 & D E & \\
SHORTCUT_1_5 & C 0 & \\
SHORTCUT_8_9 & D 0 & \\
\hline
\end{tabular}

\begin{tabular}{|llll|}
\hline \multicolumn{3}{c|}{ file.max @VISIR2 } & \\
VFGENA_FGENA1 & A 0 & $\max : 5$ & \\
VDC+25V_4 & B & $\max : 15$ & imax:0.5 \\
VDCCOM_24_2 & & 0 & \\
& & & \\
SHORTCUT_1_5 & A B & & \\
R_3 6 & C D & $10 \mathrm{~K}$ \\
SHORTCUT_1_5 & D E & & \\
R_5_2 & E F & 1K \\
SHORTCUT_8_9 & F 0 & \\
\hline
\end{tabular}

Fig. 5. Example of max file at VISIR system1 and VISIR system2. 
the other.

- FR2: Teachers and students must be able to have contents in multiple languages.

- FR3: Teachers must be able to have a single repository where to search for lessons and contents.

- FR4: Teachers must be able to take the contents and lessons and bring them to their own spaces outside PILAR using an assistant.

PILAR will seek to motivate the teachers' and students' communities external to the project to be involved in the validation activities of the project. The project resources are available to the participants and they will be helped to design a series of tuned scenarios to implement in their classes.

\section{ACKNOWLEDGMENT (Heading 5)}

The authors acknowledge the support of the Escuela de Doctorado de la UNED, the S2013/ICE-2715 - eMadrid project, VISIR + project Erasmus + Capacity Building in Higher Education $2015 \mathrm{n}^{\circ}$ 561735-EPP-1-2015-1-PTEPPKA2- CBHE-JP and PILAR project Erasmus+ Strategic Partnership $\mathrm{n}^{\circ}$ 2016-1-ES01-KA203-025327 (Platform Integration of Laboratories based on the Architecture of visiR). As well as to the Education Innovation Project (PIE) of UNED, "Desarrollos Avanzados Multi-Objetivo de Laboratorios Remotos para Actividades Educativas DAMO-LRAE", from the Vicerrectorado de Ordenación Académica y Calidad and the Instituto Universitario de Educación a Distancia (IUED) of the UNED and to the project 2018-IEQ18 from the Escuela Superior de Ingenieros Industriales of UNED.

\section{REFERENCES}

[1] "Erasmus+ Project Results - PLATFORM INTEGRATION OF LABORATORIES BASED ON THE ARCHITECTURE OF VISIR", $\begin{array}{lll}\text { Ec.europa.eu, } 2016 . & \text { [Online]. Available: }\end{array}$ http://ec.europa.eu/programmes/erasmus-plus/projects/eplus-projectdetails/\#project/2d88ecb1-3db1-4a29-93c1-dd2802eec4f6. [Accessed: 08- Jan- 2019]

[2] "European Commission - PRESS RELEASES - Press release Commission launches 'Opening up Education' to boost innovation and digital skills in schools and universities", Europa.eu, 2019. [Online]. Available: http://europa.eu/rapid/press-release_IP-13-859_en.htm. [Accessed: 09- Jan- 2019]

[3] I. Gustavsson, J. Zackrisson, J. Bartunek, H. Åkesson, L. Håkansson, and T.L. Lagö, "An instructional electronics laboratory opened for remote operation and control" International Conference on Engineering Education, 2006, San Juan (Puerto Rico).

[4] M. Tawfik et al, "Online Experiments With DC/DC Converters Using the VISIR Remote Laboratory" IEEE Rev. Iberoam. Tecnol Aprendiz., vol. 10, (4), pp. 310-318, 2015. DOI: 10.1109/RITA.2015.2486459.

[5] M. Blazquez-Merino et al., "Structured MOOC designed to optimize Electricity learning at Secondary school," 2018 IEEE Global Engineering Education Conference (EDUCON), Tenerife, 2018, pp. 223-232. doi: 10.1109/EDUCON.2018.8363232

[6] I. Gustavsson, K. Nilsson, J. Zackrisson, G.R. Alves, A.V. Fidalgo, L. Claesson, J.G. Zubia, U.H. Jayo, M. Castro, G. Diaz Orueta and F.G. Loro, "'Lab sessions in VISIR laboratories," 2016 13th International Conference on Remote Engineering and Virtual Instrumentation (REV), pp. 350-352.

[7] G.R. Alves, M.C. Viegas, M.A. Marques, M.C. Costa-Lobo, A.A. Silva, F. Formanski and J.B. Silva, '"Student performance analysis under different moodle course designs," 2012 15th International Conference on Interactive Collaborative Learning (ICL), pp. 1-5.

[8] F. Garcia-Loro, R. Fernandez, M. Gomez, H. Paz, F. Soria, M.I. Pozzo, E. Dobboletta, A. Fidalgo, G. Alves, E. Sancristobal, G. Diaz and M. Castro, '"Educational Scenarios Using Remote Laboratory VISIR for Electrical/Electronic Experimentation,", pp. 298-303.

[9] Blazquez-Merino M. et al. (2019) "Use of VISIR Remote Lab in Secondary School: Didactic Experience and Outcomes.” In: Auer M., Langmann R. (eds) Smart Industry \& Smart Education. REV 2018. Lecture Notes in Networks and Systems, vol 47. Springer, Cham

[10] F. Garcia-Loro et al., "Experimenting in PILAR federation: A common path for the future," 2018 IEEE Global Engineering Education Conference (EDUCON), Tenerife, 2018, pp. 1518-1523. doi: 10.1109/EDUCON.2018.8363413

[11] F. Garcia-Loro, et al, '" PILAR: a Federation of VISIR Remote Laboratory Systems for Educational Open Activities," 2018, TALE, Wollongong, Australia.

[12] G.R. Alves et al.., '"Spreading remote lab usage a system - A community - A Federation," 2016 2nd International Conference of the Portuguese Society for Engineering Education (CISPEE), pp. 1-7.

[13] W. Kulesza et al., "A federation of VISIR remote laboratories through the PILAR Project," 2017 4th Experiment@International Conference (exp.at'17), Faro, 2017, pp. 28-32. doi: 10.1109/EXPAT.2017.7984407 